\title{
INFLUENCE OF ORGANIC MANURE, BIOFERTILIZER AND/OR SOME VITAMIN TREATMENTS ON: A. VEGETATIVE GROWTH AND FLOWERING ASPECTS OF GLADIOLUS GRANDIFLORUS VAR. GOLD FIELD PLANTS
}

\author{
M.A.H. Abdou; M.K. Aly; A.A. El-Sayed and A.S.A. Ahmed \\ Hort. Dept., Fac. Agric., Minia Univ., Egypt
}

\begin{abstract}
A field experiment was carried out during the two successive seasons of 2016/2017 and 2017/2018. The aim of this study was to investigate the effect of farmyard manure (FYM) at four levels $\left(0,10,15\right.$ and $\left.20 \mathrm{~m}^{3} / \mathrm{fed}\right)$ in combination with Minia Azotein (M.A.) at $50 \mathrm{ml} /$ plant and/or some vitamins ( $E$ and $C$ ) each at $50 \mathrm{ppm}$, in addition to the control on vegetative growth and flowering of Gladiolus grandiflorus var. Gold Field plants. Results showed that leaf length $(\mathrm{cm})$, number of leaves/plant, leaves dry weight/plant (g), length of spike $(\mathrm{cm})$, spike diameter $(\mathrm{mm})$, spike fresh weight $(\mathrm{g})$, number of florets/spike, lower floret diameter $(\mathrm{cm})$ and lower floret fresh weight (g) were gradually increased by increasing the levels of farmyard manure with significant differences were detected between successive treatments. Also, Minia Azotein (M.A.) and/or some vitamins (E and C) treatments significantly increased all vegetative growth and flowering parameters in comparison with the control. Minia Azotein (M.A.) plus vitamins $\mathrm{E}$ and vitamin $\mathrm{C}$ were more effective in this concern. It was found also that the use of FYM $\left(20 \mathrm{~m}^{3} / \mathrm{fed}\right)$ in combination with Minia Azotein (M.A.) plus vit. E and vit. C followed by $20 \mathrm{~m}^{3} /$ fed FYM with M.A. + vit. C then $15 \mathrm{~m}^{3} /$ fed FYM with M.A. + vit. $\mathrm{E}+$ vit. C noticeably improved the different vegetative growth and flowering parameters of gladiolus plants.
\end{abstract}

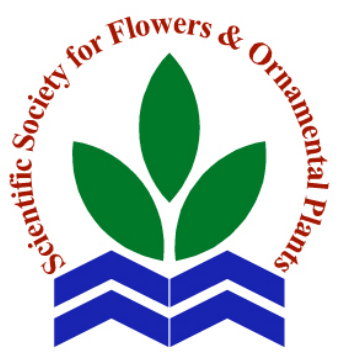

Scientific J. Flowers \& Ornamental Plants, 6(2):113-124(2019).

Received:

15/5/2019

Accepted:

30/5/2019

Key words: Gladiolus grandifloras, farmyard manure, biofertilizers, vitamins, vegetative growth, flowering.

\section{INTRODUCTION}

Gladiolus grandiflorus, L. is derived from the native plants of south and central Africa, as well as, the Mediterranean region (De-Hertogh and Le Nard, 1995). Gladiolus (Family Iridaceae) is a valuable and economic flowering bulb plant used as a landscape plant in the home gardens and in decoration as a lovely and rich colored cut flower spike with relatively long vase life (Hogan, 1990). In this study corms of Gladiolus gradiflorus var. Gold Field were chosen for their adaptability to the Egyptian environmental conditions, besides the possibility of exporting of its flowers for the demand increased.

Organic, Minia Azotein (M.A.) biofertilizer and some vitamins ( $\mathrm{E}$ and $\mathrm{C}$ ) are among the important agricultural treatments which have been proved to improve the vegetative growth and flowering aspects of gladiolus plants.

Many investigators revealed the importance of organic fertilization on the growth and flowering of gladiolus such as Gajbhiye et al. (2013), Khalil (2015), Tirkey et al. (2017), Abdou et al. (2018) and Pal and Singh (2019) on gladiolus plants, Poursafarali et al. (2011), Srivastava et al. 
(2014) and Karim et al. (2017) on tuberose plant, Mirkalae et al. (2013) and Prasad et al. (2017) on Lilium spp. and Bostan et al. (2014) on Amaryllus belladonna.

The role of Minia Azotein (M.A.) biofertilizer in improving vegetative growth and flowering parameters was revealed by Abdou et al. (2004), Hassanein and El-Sayed (2009), Kashyap (2016) and Bohra and Nautiyal (2019) on gladiolus, El-Naggar and Mahmoud (1994) on narcissus plants, Abdou (2004 a) and Abdou (2004 b) on Dahlia pinnata.

The role of alpha tocopherols (vit. E) in improving vegetative growth and flowering characters was also mentioned by Hussein et al. (2007) on cowpea, El-Lethy et al. (2010) on flax and Mohamed (2016) on Antirrhinum majus plants.

The role of ascorbic acid (vit. C) in improving vegetative growth and flowering characters was also reported by Abdel Aziz et al. (2009) and Khalil (2015) on gladiolus, Kasim and Adil (2014) on Freesia hybrid, Mohammed et al. (2016) on dahlia and Farahat et al. (2017) on Monstera delicious plants.

The aim of this work was to study the effect of FYM, Minia Azotein (M.A.) and/or some vitamins ( $E$ and $C$ ) treatments on the vegetative growth and flowering of Gladiolus grandiflorus var. Gold Field plants.

\section{MATERIALS AND METHODS}

A field experiment was carried out during the two successive seasons of 2016/2017 and 2017/2018 at the Nursery and Laboratory of Ornamental Plants, Faculty of Agriculture, Minia University to figure out the response of the Gladiolus graniflorus var. Gold Field plants to FYM, Minia Azotein (M.A.) and/or some vitamins (E and C) treatments.

The corms of gladiolus were obtained from Holland by Basiony Nurseries, Cairo, Egypt. Average corm diameter was 2.7 and
$3.3 \mathrm{~cm}$ and corm weight were 9.4 and $10.2 \mathrm{~g}$ for the first and second seasons, respectively. Corms were planted on October $1^{\text {st }}$ for both seasons in $1.5 \times 2.0 \mathrm{~m}$ plots containing 3 ridges, $50 \mathrm{~cm}$ apart. Corms were planted in hills, $20 \mathrm{~cm}$ apart (10 corms/ridge). The physical and chemical analysis of the used soil in both seasons were determined according to Jackson (1973) and shown in Table (a).

The split plot design with three replicates was followed in this experiment. The four levels of farmyard manure fertilization treatments were considered as main plots and the seven treatments of Minia Azotein (M.A.) and/or some vitamins ( $\mathrm{E}$ and C) the sub plots. The four levels of farmyard manure treatments were $0,10,15$ and 20 $\mathrm{m}^{3}$ /fed. The chemical analysis of FYM was done according to Black et al. (1965) and is shown in Table (b).

The sub plots were as follows: control, alpha tocopherols (vit. E) at 50 ppm, ascorbic acid (vit. C) at $50 \mathrm{ppm}$, Minia Azotein (M.A.) at $50 \mathrm{ml} /$ plant, M.A. + vit. E, M.A. + vit. C and M.A. + vit. E + vit. C. Vitamin $\mathrm{E}$ and $\mathrm{C}$ were sprayed three times, one month and two months after planting and after flower cut.

The following data were recorded

1. Vegetative growth characters just before flowering: leaf length $(\mathrm{cm})$, number of leaves/plant and dry weight of leaves/plant (g).

2. Flowering characters: length of spike (cm), spike diameter $(\mathrm{mm})$, spike fresh weight (g), number of florets/spike, lower floret diameter $(\mathrm{cm})$ and lower floret fresh weight (g).

Data of the two experiments were subjected to the statistical analysis of variance using MSTAT-C (1986). L.S.D. test at $5 \%$ was used to compare the means of treatments. 
Table a. Physical and chemical properties of the experimental soil.

\begin{tabular}{|c|c|c|c|c|c|c|}
\hline \multirow{2}{*}{ Soil character } & \multicolumn{2}{|c|}{ Values } & \multirow{2}{*}{\multicolumn{2}{|c|}{ Soil character }} & \multicolumn{2}{|c|}{ Values } \\
\hline & $2016 / 2017$ & $2017 / 2018$ & & & $2016 / 2017$ & $2017 / 2018$ \\
\hline Sand \% & 28.20 & 28.98 & \multicolumn{2}{|c|}{ Available P \% } & 15.15 & 15.64 \\
\hline Silt \% & 30.70 & 29.87 & \multicolumn{2}{|c|}{ Exchangeable $\mathbf{K}^{+}$} & 2.09 & 2.82 \\
\hline Clay \% & 41.10 & 41.15 & \multicolumn{2}{|c|}{ Exch. $\mathrm{Ca}^{++} \mathrm{mg} / 100 \mathrm{~g}$ soil } & 31.71 & 31.10 \\
\hline Soil texture & Clay loam & Clay loam & \multicolumn{2}{|c|}{ Exch. $\mathrm{Na}^{+} \mathrm{mg} / \mathbf{1 0 0}$ g soil } & 2.42 & 2.53 \\
\hline Organic matter \% & 1.59 & 1.56 & & & & \\
\hline $\mathrm{CaCO}_{3} \%$ & 2.08 & 2.10 & & $\mathbf{F e}$ & 8.52 & 8.22 \\
\hline pH 1:2.5 & 7.80 & 7.77 & DTPA & $\mathbf{C u}$ & 2.05 & 2.02 \\
\hline E.C. m mhose/cm & 1.06 & 1.07 & Ext. ppm & Zn & 2.74 & 2.85 \\
\hline Total N \% & 0.07 & 0.08 & & Mn & 8.24 & 8.09 \\
\hline
\end{tabular}

Table b. Chemical analysis of FYM applied in the present study.

\begin{tabular}{lcclcc}
\hline Properties & $\mathbf{1}^{\text {st }}$ season & $\mathbf{2}^{\text {nd }}$ season & \multicolumn{1}{c}{ Properties } & $\mathbf{1}^{\text {st }}$ season & $\mathbf{2}^{\text {nd }}$ season \\
\hline Organic matter \% & 28.0 & 27.5 & K \% & 1.18 & 1.22 \\
Carbon \% & 15.80 & 16.65 & Fe ppm & 239.0 & 237.5 \\
Total N \% & 0.92 & 0.95 & Zn ppm & 271.2 & 273.1 \\
C/N ratio & 17.17 & 17.53 & Mn ppm & 233.5 & 235.8 \\
Humidity \% & 8.00 & 7.91 & pH & 7.31 & 7.21 \\
P \% & 0.23 & 0.25 & E.C. (mhose/cm) & 1.07 & 1.08 \\
\hline
\end{tabular}

\section{RESULTS AND DISCUSSION}

\section{Vegetative growth characters:}

Data presented in Table (1) showed that leaf length (cm), number of leaves/plant and leaves dry weight/plant (g) of gladiolus were significantly increased, in both seasons, due to the use of 10,15 and $20 \mathrm{~m}^{3} /$ fed FYM in comparison with those of control plants. Moreover, the increase was gradually by the gradual increase in FYM level. The increase in vegetative growth traits due to FYM at levels of 10,15 and $20 \mathrm{~m}^{3} /$ fed over the control reached 9.18, 15.57 and $23.30 \%$ for leaf length, 4.11, 7.59 and $11.08 \%$ for leaf number and 30.67, 48.67 and $65.67 \%$ for leaves dry weight, respectively, in the first season and by 2.89, 12.70 and $18.94 \%$ for leaf length, 12.24, 21.13 and $28.87 \%$ for number of leaves and 13.29, 21.45 and 30.51 for leaves dry weight, respectively in the second one. Similar results were found by Pandey et al. (2013), Abdou and Ibrahim (2015), Abdou et al. (2018) and Pal and Singh (2019) on gladiolus, Treder (2008) and El-Naggar and El-Nasharty (2009) on
Hippeastrum vittatum, Moghadam et al. (2012) on lily, Pandey et al. (2017) on dahlia, Abduallah (2019) on Iris and Samoon et al. (2019) on Alstroemeria plant. The increase of vegetative growth resulting from using FYM as organic fertilization might be due to that organic matter is considered an important factor for improving the physical, chemical and biological properties of the soil and consequently, increasing plant growth (Munne-Bosch and Algere, 2002).

Data presented in Table (1) indicated that, leaf length, number of leaves/plant and leaves dry weight of gladiolus were significantly increased, in both seasons, due to the use of Minia Azotein (M.A.) and/or some vitamins ( $\mathrm{E}$ and $\mathrm{C}$ ), each at $50 \mathrm{ppm}$ either used separately or together in comparison with control. The combined treatments of Minia Azotein (M.A.) plus vitamin $\mathrm{E}$ plus vitamin $\mathrm{C}$ seemed to be more effective than other treatments. In conformity with these results were those detected by Taha and Hassan (2008), Mazhar and Eid (2016), Pansuriya et al. (2018) 
Table 1. Effect of farmyard manure (FYM), biofertilizer and/or some vitamin treatments, as well as, their combination treatments on leaf length, number of leaves/plant (cm) and leaves dry weight (g) of Gladiolus grandiflorus var. Gold Field during the first and second seasons.

Biofertilization and some vitamin treatments (B)

FYM levels (m³/fed) (A)

$$
\begin{array}{llllclcccc} 
& \multicolumn{3}{c}{1^{\text {st }} \text { season }(2016 / 2017)} & \multicolumn{5}{c}{2^{\text {nd }} \text { season }(2017 / 2018)} \\
0 & 10 & 15 & 20 & \begin{array}{c}
\text { Mean } \\
\text { (B) }
\end{array} & 0 & 10 & 15 & 20 & \text { Mean } \\
\end{array}
$$

Leaf length $(\mathrm{cm})$

\begin{tabular}{lcccccccccc}
\hline Control & 45.62 & 49.54 & 53.16 & 57.21 & 51.38 & 44.67 & 44.67 & 48.67 & 51.00 & 47.25 \\
Alpha tocopherol (vit. E) & 48.71 & 52.23 & 56.02 & 60.00 & 54.24 & 47.00 & 47.00 & 52.33 & 53.00 & 49.83 \\
Ascorbic acid (vit. C) & 51.87 & 55.66 & 59.24 & 63.14 & 57.48 & 47.67 & 49.33 & 53.33 & 56.67 & 51.75 \\
Minia Azotein (M.A.) & 54.50 & 58.40 & 61.69 & 66.02 & 60.15 & 50.33 & 50.67 & 55.00 & 58.33 & 53.58 \\
M.A. + vit. E & 55.25 & 61.50 & 64.63 & 69.07 & 62.61 & 51.67 & 53.33 & 58.33 & 60.33 & 55.92 \\
M.A. + vit. C & 57.79 & 64.50 & 67.74 & 72.02 & 65.51 & 52.00 & 55.00 & 59.33 & 65.00 & 57.83 \\
M.A. + vit. E + vit. C & 61.24 & 67.59 & 70.88 & 74.91 & 68.66 & 53.33 & 56.67 & 63.67 & 68.00 & 60.42 \\
Mean (A) & 53.57 & 58.49 & 61.91 & 66.05 & & 49.52 & 50.95 & 55.81 & 58.90 &
\end{tabular}

L.S.D. at 5 \%

A: 1.18

AB: 2.10

A: 1.41

B: 1.62

AB: 3.24

Number of leaves/plant

$\begin{array}{lcccccccccc}\text { Control } & 5.96 & 6.08 & 6.30 & 6.58 & 6.23 & 7.05 & 8.05 & 8.57 & 9.57 & 8.31 \\ \text { Alpha tocopherol (vit. E) } & 6.04 & 6.21 & 6.57 & 6.73 & 6.39 & 7.55 & 8.55 & 9.55 & 10.08 & 8.93 \\ \text { Ascorbic acid (vit. C) } & 6.10 & 6.46 & 6.72 & 6.83 & 6.53 & 8.10 & 9.15 & 10.06 & 10.72 & 9.51 \\ \text { Minia Azotein (M.A.) } & 6.37 & 6.71 & 6.82 & 6.93 & 6.71 & 9.05 & 10.05 & 10.57 & 11.11 & 10.20 \\ \text { M.A. + vit. E } & 6.42 & 6.79 & 6.88 & 7.21 & 6.83 & 9.13 & 10.22 & 11.06 & 11.73 & 10.54 \\ \text { M.A. + vit. C } & 6.62 & 6.87 & 7.04 & 7.37 & 6.98 & 9.59 & 10.84 & 11.55 & 12.22 & 11.05 \\ \text { M.A. + vit. E + vit. C } & 6.75 & 6.96 & 7.29 & 7.46 & 7.12 & 10.16 & 11.21 & 12.05 & 12.72 & 11.54 \\ \text { Mean (A) } & 6.32 & 6.58 & 6.80 & 7.02 & & 8.66 & 9.72 & 10.49 & 11.16 & \end{array}$

L.S.D. at $5 \%$

A: 0.19

B: 0.04

AB: 0.08

A: 0.26

B: 0.09

AB: 0.18

\section{Leaves dry weight (g)}

\begin{tabular}{lllllllllll} 
Control & 1.16 & 2.70 & 3.01 & 3.69 & 2.64 & 2.65 & 2.97 & 3.35 & 3.72 & 3.17 \\
Alpha tocopherol (vit. E) & 2.29 & 2.93 & 3.67 & 4.13 & 3.26 & 2.78 & 3.29 & 3.62 & 3.88 & 3.39 \\
Ascorbic acid (vit. C) & 2.81 & 3.63 & 4.05 & 4.66 & 3.79 & 3.19 & 3.56 & 3.84 & 4.10 & 3.67 \\
Minia Azotein (M.A.) & 3.12 & 3.96 & 4.55 & 4.90 & 4.13 & 3.36 & 3.82 & 3.99 & 4.35 & 3.88 \\
M.A. + vit. E & 3.45 & 4.35 & 4.84 & 5.35 & 4.50 & 3.49 & 3.96 & 4.25 & 4.54 & 4.06 \\
M.A. + vit. C & 3.90 & 4.70 & 5.30 & 5.87 & 4.94 & 3.76 & 4.22 & 4.50 & 4.70 & 4.30 \\
M.A. + vit. E + vit. C & 4.27 & 5.18 & 5.81 & 6.18 & 5.36 & 3.93 & 4.46 & 4.61 & 4.96 & 4.49 \\
Mean (A) & 3.00 & 3.92 & 4.46 & 4.97 & & 3.31 & 3.75 & 4.02 & 4.32 & \\
L.S.D. at 5 \% & A: 0.46 & B: 0.34 & AB: 0.68 & A: 0.26 & B: 0.18 & AB: 0.36 \\
\hline
\end{tabular}


and Bohra and Nautiyal (2019) on gladiolus, El-Naggar and Mahmoud (1994) on narcissus, Abdou (2004) on dahlia and Attia et al. (2018) on tuberose plant regarding the effect of biofertilizer. Moreover, Abdou et al. (2012) on mint, Ibrahim (2014) on khella and Mohamed (2016) on Antirrhinum majus plant concerning the influence of vitamin $\mathrm{E}$. Also, Abdel Aziz et al. (2009), Abo Leila and Eid (2011) and Khalil (2015) on gladiolus, Kasim and Adil (2014) on Freesia, Mohammed et al. (2016) on dahlia and Gaber (2019) on Pelargonium zonale plant concerning the effect of vitamin $\mathrm{C}$ attained similar results.

Application of active biofertilizer (Minia Azotein "M.A.") as a commercial biofertilizers stimulate containing N-fixing bacteria on gladiolus plants. Many investigators explained the role of $\mathrm{N}$-fixing bacteria (Jagnow et al., 1991; Hauwaka, 2000 and Gadagi et al., 2004).

The most important function of $\alpha$ tocopherol is to act as recyclable chain reaction terminators of polyunsaturated fatty acids, free radicals generated by lipids oxidation (Munne-Bosch and Algere, 2002).

Ascorbic acid is the most abundant antioxidant which protects plant cells, or antioxidant defense, photoprotection and regulation of photosynthesis and growth (Dewick, 2000).

The interaction between FYM, Minia Azotein and/or some vitamin treatments was significant in the two seasons for all previous characters. The maximum values were obtained due to fertilizing the soil of gladiolus with high level of FYM (20 $\mathrm{m}^{3} /$ fed) and adding Minia Azotein biofertilizer at the rate of $50 \mathrm{ml} / \mathrm{hill}$ and spraying plants with vitamin E (50 ppm) + vitamin C (50 ppm).

\section{Flowering parameters:}

Data presented in Tables (2 and 3) indicated that all FYM levels caused significant increases in length of spike $(\mathrm{cm})$, spike diameter (mm), spike fresh weight (g), number of florets/spike, lower floret diameter $(\mathrm{cm})$ and lower floret fresh weight (g), in the two seasons, in comparison with those of untreated plants. The means of flowering parameters were gradually increased according to the increase in levels of farmyard manure fertilizer. The application of FYM at high level $\left(20 \mathrm{~m}^{3} / \mathrm{fed}\right)$ resulted the highest values of various flowering traits. These results are in close with those obtained by Atta-Alla et al. (2003), Khalil (2015) and Baruati et al. (2018) on gladiolus, Abass (2003), AbdelSattar et al. (2010), Srivastava et al. (2014) and Karim et al. (2017) on tuberose, ElNaggar and El-Nasharty (2009) on Hippeastrum vittatum.

A possible explanation to the positive effect of FYM fertilizer treatments might be attributed to its stimulative effects on different vegetative growth (Tables, 2 and 3). Better vegetative growth should be directly reflected on various flowering aspects.

Regarding the effect of Minia Azotein biofertilizer and/or vitamin (E and C) treatments, data presented in Tables (2 and 3) revealed that all six treatments significantly increased length of spike $(\mathrm{cm})$, spike diameter (mm), spike fresh weight (g), number of florets/spike, lower floret diameter $(\mathrm{cm})$ and lower floret fresh weight (g) compared with untreated plants. The highest values for all flowering characters were obtained due to gladiolus plants inoculated with Minia Azotein (M.A.) biofertilizer plus spraying with alpha tocopherol and ascorbic acid each at $50 \mathrm{ppm}$ in both seasons.

This finding was similar to those obtained by Pansuriya et al. (2018) on gladiolus regarding the influence of Minia Azotein biofertilizer treatment. Moreover, Khalil (2015) on gladiolus regarding the effects of vitamin E. Also, Abdel Aziz et al. (2009) and Khalil (2015) on gladiolus plant regarding the influence of vitamin $\mathrm{C}$.

The interaction treatments exhibited a significant effect on all flowering parameter. The highest values were obtained with FYM 
Table 2. Effect of farmyard manure (FYM), biofertilizer and/or some vitamin treatments, as well as, their combination treatments on length of spike $(\mathrm{cm})$, spike diameter (mm) and spike fresh weight (g) of Gladiolus grandiflorus var. Gold Field during the first and second seasons.

\begin{tabular}{|c|c|c|c|c|c|c|c|c|c|c|}
\hline \multirow{3}{*}{$\begin{array}{l}\text { Biofertilization } \\
\text { and some vitamin } \\
\text { treatments (B) }\end{array}$} & \multicolumn{10}{|c|}{ FYM levels (m³/fed) (A) } \\
\hline & \multicolumn{5}{|c|}{$1^{\text {st }}$ season $(2016 / 2017)$} & \multicolumn{5}{|c|}{$2^{\text {nd }}$ season $(2017 / 2018)$} \\
\hline & $\mathbf{0}$ & 10 & 15 & 20 & $\begin{array}{c}\text { Mean } \\
\text { (B) }\end{array}$ & $\mathbf{0}$ & 10 & 15 & 20 & $\begin{array}{c}\text { Mean } \\
\text { (B) }\end{array}$ \\
\hline \multicolumn{11}{|c|}{ Length of spike (cm) } \\
\hline Control & 45.79 & 53.58 & 54.08 & 55.22 & 52.17 & 60.32 & 63.17 & 65.31 & 67.82 & 64.16 \\
\hline Alpha tocopherol (vit. E) & 53.04 & 53.96 & 54.71 & 56.23 & 54.49 & 61.01 & 63.32 & 67.55 & 69.50 & 65.35 \\
\hline Ascorbic acid (vit. C) & 53.66 & 54.53 & 56.04 & 57.33 & 55.39 & 63.29 & 67.47 & 69.39 & 72.04 & 68.05 \\
\hline Minia Azotein (M.A.) & 54.17 & 55.87 & 57.26 & 59.12 & 56.61 & 65.57 & 68.98 & 71.14 & 73.00 & 69.67 \\
\hline M.A. + vit. E & 54.31 & 57.20 & 58.79 & 60.96 & 57.82 & 67.44 & 70.21 & 72.17 & 74.07 & 70.97 \\
\hline M.A. + vit. C & 55.25 & 58.29 & 59.95 & 61.79 & 58.82 & 68.63 & 72.10 & 73.81 & 75.80 & 72.59 \\
\hline M.A. + vit. E + vit. C & 56.49 & 59.37 & 61.33 & 61.81 & 59.75 & 69.85 & 73.35 & 75.16 & 76.10 & 73.62 \\
\hline Mean (A) & 53.24 & 56.11 & 57.45 & 58.92 & & 65.16 & 68.37 & 70.65 & 72.62 & \\
\hline L.S.D. at $5 \%$ & \multicolumn{2}{|c|}{ A: 1.13} & B: 0.55 & \multicolumn{2}{|c|}{ AB: 1.10} & \multicolumn{2}{|c|}{ A: 1.24} & B: 1.02 & \multicolumn{2}{|c|}{ AB: 2.04} \\
\hline \multicolumn{11}{|c|}{ Spike diameter (mm) } \\
\hline Control & 0.51 & 0.53 & 0.59 & 0.65 & 0.57 & 0.40 & 0.43 & 0.48 & 0.53 & 0.46 \\
\hline Alpha tocopherol (vit. E) & 0.52 & 0.58 & 0.64 & 0.69 & 0.61 & 0.42 & 0.45 & 0.52 & 0.57 & 0.49 \\
\hline Ascorbic acid (vit. C) & 0.57 & 0.62 & 0.68 & 0.73 & 0.65 & 0.44 & 0.51 & 0.56 & 0.62 & 0.53 \\
\hline Minia Azotein (M.A.) & 0.60 & 0.67 & 0.72 & 0.76 & 0.69 & 0.49 & 0.55 & 0.61 & 0.67 & 0.58 \\
\hline M.A. + vit. E & 0.61 & 0.71 & 0.75 & 0.81 & 0.72 & 0.50 & 0.60 & 0.66 & 0.79 & 0.64 \\
\hline M.A. + vit. C & 0.66 & 0.74 & 0.78 & 0.84 & 0.76 & 0.54 & 0.65 & 0.73 & 0.83 & 0.69 \\
\hline M.A. + vit. E + vit. C & 0.70 & 0.77 & 0.83 & 0.86 & 0.79 & 0.58 & 0.68 & 0.81 & 0.86 & 0.73 \\
\hline Mean (A) & 0.60 & 0.66 & 0.71 & 0.76 & & 0.48 & 0.55 & 0.62 & 0.70 & \\
\hline L.S.D. at $5 \%$ & \multicolumn{2}{|c|}{ A: 0.03} & B: 0.02 & \multicolumn{2}{|c|}{ AB: 0.04} & \multicolumn{2}{|c|}{ A: 0.05} & B: 0.03 & \multicolumn{2}{|c|}{ AB: 0.06} \\
\hline \multicolumn{11}{|c|}{ Spike fresh weight (g) } \\
\hline Control & 7.47 & 7.77 & 8.26 & 10.03 & 8.38 & 11.82 & 14.02 & 16.54 & 18.01 & 15.10 \\
\hline Alpha tocopherol (vit. E) & 7.71 & 8.18 & 9.74 & 10.89 & 9.13 & 13.05 & 16.13 & 17.82 & 19.09 & 16.52 \\
\hline Ascorbic acid (vit. C) & 7.87 & 9.15 & 10.60 & 11.62 & 9.81 & 15.28 & 17.31 & 18.89 & 20.37 & 17.96 \\
\hline Minia Azotein (M.A.) & 8.81 & 10.42 & 11.54 & 12.56 & 10.83 & 17.17 & 18.31 & 20.24 & 21.14 & 19.22 \\
\hline M.A. + vit. E & 9.08 & 11.40 & 12.39 & 13.63 & 11.63 & 17.19 & 19.72 & 21.04 & 22.00 & 19.99 \\
\hline M.A. + vit. C & 10.37 & 12.38 & 13.37 & 14.57 & 12.67 & 18.03 & 20.84 & 21.54 & 23.35 & 20.94 \\
\hline M.A. + vit. E + vit. C & 11.31 & 12.80 & 14.37 & 14.63 & 13.28 & 19.45 & 21.20 & 22.84 & 23.55 & 21.76 \\
\hline Mean (A) & 8.95 & 10.30 & 11.47 & 12.56 & & 16.00 & 18.22 & 19.84 & 21.07 & \\
\hline L.S.D. at $5 \%$ & \multicolumn{2}{|c|}{ A: 1.08} & B:0.40 & \multicolumn{2}{|c|}{ AB: 0.80} & \multicolumn{2}{|c|}{ A: 1.21} & B: 0.75 & \multicolumn{2}{|c|}{ AB: 1.50} \\
\hline
\end{tabular}


Table 3. Effect of farmyard manure (FYM), biofertilizer and/or some vitamin treatments, as well as, their combination treatments on number of florets/spike, lower floret diameter and lower floret fresh weight (g) of Gladiolus grandiflorus var. Gold Field during the first and second seasons.

\begin{tabular}{|c|c|c|c|c|c|c|c|c|c|c|}
\hline \multirow{3}{*}{$\begin{array}{l}\text { Biofertilization } \\
\text { and some vitamin } \\
\text { treatments (B) }\end{array}$} & \multicolumn{10}{|c|}{ FYM levels (m³/fed) (A) } \\
\hline & \multicolumn{5}{|c|}{$1^{\text {st }}$ season $(2016 / 2017)$} & \multicolumn{5}{|c|}{$2^{\text {nd }} \operatorname{season}(2017 / 2018)$} \\
\hline & $\mathbf{0}$ & 10 & 15 & 20 & $\begin{array}{c}\text { Mean } \\
\text { (B) }\end{array}$ & $\mathbf{0}$ & 10 & 15 & 20 & $\begin{array}{c}\text { Mean } \\
\text { (B) }\end{array}$ \\
\hline \multicolumn{11}{|c|}{ Number of florets/spike } \\
\hline Control & 6.72 & 7.15 & 7.43 & 7.75 & 7.26 & 7.51 & 7.83 & 8.36 & 8.67 & 8.09 \\
\hline Alpha tocopherol (vit. E) & 7.12 & 7.41 & 7.73 & 8.29 & 7.64 & 7.62 & 8.31 & 8.66 & 8.94 & 8.38 \\
\hline Ascorbic acid (vit. C) & 7.40 & 7.71 & 8.16 & 8.54 & 7.95 & 8.00 & 8.54 & 8.93 & 9.32 & 8.70 \\
\hline Minia Azotein (M.A.) & 7.46 & 8.08 & 8.50 & 8.89 & 8.23 & 8.40 & 8.87 & 9.28 & 9.55 & 9.03 \\
\hline M.A. + vit. E & 7.57 & 8.46 & 8.68 & 9.21 & 8.48 & 8.46 & 9.06 & 9.51 & 9.93 & 9.24 \\
\hline M.A. + vit. C & 7.87 & 8.66 & 8.97 & 9.50 & 8.75 & 8.78 & 9.49 & 9.79 & 10.08 & 9.54 \\
\hline M.A. + vit. E + vit. C & 8.45 & 8.95 & 9.44 & 9.91 & 9.19 & 9.00 & 9.57 & 9.98 & 10.33 & 9.72 \\
\hline Mean (A) & 7.51 & 8.06 & 8.42 & 8.87 & & 8.25 & 8.81 & 9.22 & 9.55 & \\
\hline L.S.D. at $5 \%$ & \multicolumn{2}{|c|}{ A: 0.43} & B:0.24 & \multicolumn{2}{|c|}{ AB:0.48 } & \multicolumn{2}{|c|}{ A: 0.31} & B: 0.18 & \multicolumn{2}{|c|}{ AB: 0.36} \\
\hline \multicolumn{11}{|c|}{ Lower floret diameter $(\mathbf{c m})$} \\
\hline Control & 6.48 & 7.04 & 7.32 & 7.71 & 7.14 & 6.50 & 7.14 & 7.30 & 7.84 & 7.20 \\
\hline Alpha tocopherol (vit. E) & 6.94 & 7.16 & 7.68 & 7.88 & 7.42 & 6.88 & 7.29 & 7.78 & 8.13 & 7.52 \\
\hline Ascorbic acid (vit. C) & 7.09 & 7.55 & 7.86 & 8.15 & 7.66 & 7.16 & 7.65 & 7.93 & 8.19 & 7.73 \\
\hline Minia Azotein (M.A.) & 7.37 & 7.84 & 8.10 & 8.28 & 7.90 & 7.46 & 7.92 & 8.17 & 8.51 & 8.02 \\
\hline M.A. + vit. E & 7.47 & 8.09 & 8.22 & 8.47 & 8.06 & 7.55 & 8.16 & 8.47 & 8.73 & 8.23 \\
\hline M.A. + vit. C & 7.74 & 8.16 & 8.33 & 8.53 & 8.19 & 7.85 & 8.39 & 8.71 & 8.77 & 8.43 \\
\hline M.A. + vit. E + vit. C & 7.93 & 8.30 & 8.51 & 8.71 & 8.36 & 8.15 & 8.67 & 8.75 & 8.98 & 8.64 \\
\hline Mean (A) & 7.29 & 7.73 & 8.00 & 8.25 & & 7.36 & 7.89 & 8.16 & 8.45 & \\
\hline L.S.D. at $5 \%$ & \multicolumn{2}{|c|}{ A: 0.20} & B: 0.09 & \multicolumn{2}{|c|}{ AB: 0.18} & \multicolumn{2}{|c|}{ A: 0.23} & B: 0.11 & \multicolumn{2}{|c|}{ AB: 0.22} \\
\hline \multicolumn{11}{|c|}{ Lower floret fresh weight (g) } \\
\hline Control & 3.16 & 3.45 & 3.51 & 3.84 & 3.49 & 3.22 & 3.51 & 3.58 & 3.83 & 3.54 \\
\hline Alpha tocopherol (vit. E) & 3.40 & 3.48 & 3.81 & 3.99 & 3.67 & 3.33 & 3.56 & 3.81 & 4.04 & 3.69 \\
\hline Ascorbic acid (vit. C) & 3.46 & 3.76 & 3.87 & 4.10 & 3.80 & 3.52 & 3.77 & 3.98 & 4.28 & 3.89 \\
\hline Minia Azotein (M.A.) & 3.60 & 3.86 & 4.05 & 4.22 & 3.93 & 3.69 & 3.95 & 4.27 & 4.47 & 4.10 \\
\hline M.A. + vit. E & 3.74 & 4.02 & 4.19 & 4.72 & 4.17 & 3.71 & 4.25 & 4.46 & 4.73 & 4.29 \\
\hline M.A. + vit. C & 3.85 & 4.12 & 4.30 & 4.83 & 4.28 & 3.91 & 4.41 & 4.56 & 5.04 & 4.48 \\
\hline M.A. + vit. E + vit. C & 4.01 & 4.26 & 4.76 & 5.17 & 4.55 & 4.09 & 4.48 & 4.77 & 5.19 & 4.63 \\
\hline Mean (A) & 3.60 & 3.85 & 4.07 & 4.41 & & 3.64 & 3.99 & 4.20 & 4.51 & \\
\hline L.S.D. at $5 \%$ & \multicolumn{2}{|c|}{ A: 0.21} & B: 0.17 & \multicolumn{2}{|c|}{ AB: 0.34} & \multicolumn{2}{|c|}{ A: 0.18} & B: 0.15 & \multicolumn{2}{|c|}{ AB: 0.30} \\
\hline
\end{tabular}


at $20 \mathrm{~m}^{3} / \mathrm{fed}$ in combination with Minia Azotein biofertilizer plus vitamin $\mathrm{E}+$ vitamin C.

\section{REFERENCES}

Abass, R.A. (2003). Response of Polianthus tuberosa, L. to sewage sludge addition at the newly reclaimed soils. Alex Sci. Exch., 24: 283-297.

Abdel Aziz, G.N.; Taha, L. and Ibrahim, S.M.M. (2009). Some studies on the effect of putrescine, ascorbic acid and thiamine on growth, flowering and some chemical constituents of gladiolus at Nubaria. Ozean J. of Applied Sci., 2(2):169-179.

Abdel-Sattar, M.M.; Ahmed, S.S. and Nabih, A. (2010). Response of tuberose (Polianthus tuberosa, L.) plant to different soil mixtures and ethephon under reclaimed desert soil. Egypt. J. Biotechnol., 35:185-206.

Abdou, M.A.H. (2004a). Physiological studies on dahlia I- Effect of N-fixing biofertilization and growth regulators on growth, flowering and chemical constituents of Dahlia pinnata, DAHL. cv. Moonlight Sonata plants. Minia J. of Agric. Res. \& Develop., 24(3):415-436.

Abdou, M.A.H. (2004b). Physiological studies on dahlia II- Effect of chemical and biofertilization treatments of Dahlia pinnata, DAHL. cv. Moonlight Sonata plants grown in sandy calcareous soil. Minia J. of Agric. Res. \& Develop., 24(3):437-456.

Abdou, M.A.H. and Ibrahim, T.I.E. (2015). Response of gladiolus cv. Carmen to compost, biofertilization and some vitamin treatments. Proc. $1^{\text {st }}$ Conf. of SSFOP "Future of Ornamental Plants in Egypt”, Scientific J. Flowers \& Ornamental Plants, 2(1):1-10.

Abdou, M.A.H.; Attia, F.A.; Aly, M.K. and Sayed, I.H. (2004). Response of gladiolus plants to some bio and chemical fertilization treatments. 1- Vegetative growth and flowering; Proc. fifth Arabian
Hort. Con. Ismailia, Egypt, 24-28 March, 1:50-62.

Abdou, M.A.H.; Attia, F.A.; Ahmed, E.T.; El-Sayed, A.A. and Abd-El-Naeem L.M. (2012). Physiological studies on mint plants. Proc. Second Inter. Conf. of Physiol. Microb. and Ecological Plant Sci. (29-30 April, 2012) Fac. Science, Minia Univ., pp: 207-228.

Abdou, M.A.H.; Badran, F.S.; Ahmed, E.T.; Taha, R.A. and Abdel-Mola, M.A.M. (2018). Effect of compost and some natural stimulant treatments on: I. Vegetative growth and flowering aspects of (Gladiolus grandiflorus cv. Peter Pears) plants. Proc. $4^{\text {th }}$ Conf. of SSFOP "Ornamental Plants and Environment", Scientific J. Flowers \& Ornamental Plants, 5(2):105-114.

Abduallah, A.M.A. (2019). Effect of compost, Potassium Silicate and Amino Acids on Iris Plants. M.Sc. Thesis, Fac. Agric., Minia Univ., Egypt.

Abo Leila, B. and Eid, R. (2011). Improving gladiolus growth, flower keeping quality by using some vitamins application. Journal of American Science, 7(3):169174.

Atta-Alla, H.K.; Zaghloul, M.A. and Hashish, K.H. (2003). Effect of organic manure and NPK fertilizers on vegetative growth, flowering and chemical composition of some gladiolus cultivars. Annals Agric. Sci., Moshtohor, 41(2):680-691.

Attia, K.E.; Elbohy, N.F.S.; Ashour, N.A.M. (2018):Response of tuberose plants (Polianthes tuberosa, L.) to chemical and bio fertilization and their effect on vegetative growth, flowering and chemical composition under sandy soil conditions. Scientific J. Flowers \& Ornamental Plants, 5(3):261-273.

Baruati, D.; Talukdar, M.C. and Kumar, V. (2018). Effect of organic manures and biofertilizers on growth and yield of gladiolus (Gladiolus grandiflorus, L.). 
International Journal of Chemical Studies. 6(5):2529-2532.

Black, C.A.; Evans, D.D.; Evsminger, L.E.; White, J.L. and Clark, F.E. (1965). Methods of Soils Analysis. American Society of Agronomy. Inc. Publ. Madison, Wisconsin, USA, pp: 11621168.

Bohra, M. and Nautiyal, B.P. (2019). Effect of plant density and INM on vegetative, floral and yield attributes of gladiolus (Gladiolus grandiflorus, L.). Agricultural Reviews, 40(1):45-52.

Bostan, N.; Sajid, M.; Wahid, F.; Rabi, F.; Qureshi, S.; Ahmad, W.; Ahmad, S. and Tawab, S. (2014). Effect of growing media and irrigation interval on growth of Amaryllus (Amaryllus belladonna). Advances in Life Science and Technology, 18:20-27.

De-Hertogh, A. and Le Nard, M. (1995). Botanical aspects of flower bulbs. In the Physiology of Flower Bulbs, El-Sevier Amsterdam, London, New York, Tokyo, pp: 7-33.

Dewick, P.M. (2000). Medicinal Natural Products. A Biosynthetic Approach $2^{\text {nd }}$ Ed., John Wiley and Sons, N.Y., pp: 306356.

El-Lethy, S.R.; Ayad, H.S and Talaat, I.M. (2010). Physiological effect of some antioxidants on flax plant (Linum usitatissimum, L.). World Journal of Agriculture Sciences, 6(5):622-629.

El-Naggar, A.H. and El-Nasharty, A.B. (2009). Effect of growing media and mineral fertilization on growth, flowering, bulbs productivity and chemical constituents of Hippeatrum vittatum, Herb. American-Eurasian J. Agric. and Enviro. Sci., 6(3):360-371.

El-Naggar, A.L. and Mahmoud, S.M. (1994). Effect of inoculation with certain Azospirillum strains and nitrogen fertilization on Narcissus tazetta, L. under different soil textures. Assiut J. Sci., 25 (4):135-151.
Farahat, M.M.; Abdel Aziz, N.G.; Hashish, K.I. and Mazhar, A.A.M. (2017). Effect of ascorbic acid on growth and chemical constituents of Monstera delicious under lead pollutant conditions. Agricultural Engineering International Journal, 1(1):239-244.

Gaber, K.M. (2019). Vegetative and flowering growth of geranium as affected by mineral fertilization and ascorbic acid foliar application. Middle East J. Appl. Sci., 9(1):220-230.

Gadagi, R.S.; Krishnaraj, P.U. ; Kulkarni, J. H. and Tongmin, Sa. (2004). The effect of combined Azospirillum inoculation and nitrogen fertilizer on plant growth promotion and yield response of the blanket flower (Gaillardia pulchella). Scientia Horticulture, 100:323-332.

Gajbhiye, B.R.; Vetal, R.A.; Puri, A.N. and Adsul, P.B. (2013). Effect of FYM, N, P and $\mathrm{K}$ levels on growth and flowering of gladiolus (Gladiolus grandiflorus) cv. White Prosperity. Journal of Rural and Agricultural Research, 13(2):94-97.

Hassanein, M.M. and El-Sayed, S.G. (2009). Effect of some organic and biofertilization treatments on gladiolus plants: 1- Vegetative growth and flowering. J. Agric. Sci. Mansoura Univ., 34(6):6237-6254.

Hauwaka, F.I.A. (2000). Effect of using single and composite inoculation with Azospirillum brasilense, Bacillus megatherium var. phosphaticum and Glomus marcocarpus for improving growth of Zea mays. J. Agric. Sci. Mansoura Univ., 25(1):239-252.

Hogan, L. (1990). Bulbs for all Seasons. Sunset Western Garden Book, Menlo Park, CA: Lane Publishing, California, pp: 185-198.

Hussein, M.M.; Balba, L.K. and Gaballah, M.S. (2007). Developing a salt tolerant cowpea using $\alpha$-tocophrerol. Journal of Applied Sciences Research, 3(10):12341239. 
Ibrahim, T.I.E.I. (2014). Influence of Some Agricultural Treatments on Ammi visnaga Plants. Ph.D. Thesis, Fac. Agric., Minia Univ., Egypt.

Jackson, M.L. (1973). Soil Chemical Analysis Englewood Cliffs., New Prentice-Hall INC., New York.

Jagnow, G.; Hoflich, G. and Hoffman, K.H. (1991). Inoculation of non-symbiotic rhizosphere bacteria: Possibilities of increasing and stability yield. A new Botanik, 65:97-126.

Karim, K.P.; Kumar, N.V.; Raghupati, B. and Pal, A.K. (2017). Effect of biostimulants on growth and floral attributes of tuberose (Polianthes tuberosa, L.) cv. Prajwal. Int. J. Curr. Microbiol. App. Sci., 6(6):2557-2564.

Kashyap, S.K. (2016). Effect of Biofertilizers With Different Levels of Nitrogen and Phosphorus on Growth and Flower Yield of Gladiolus (Gladiolus grandiflorus, L.). M.Sc. (Hort.) Thesis, Floriculture \& Landscape Architecture Dept. India.

Kasim, J.Y. and Adil, A.M. (2014). Effect of gibberellic acid, spraying micronutrient and ascorbic acid on the vegetative growth and flowering of Freesia hybrida cV. Prominence. Journal of Kirkuk University for Agricultural Sciences, 5 (1):50-64.

Khalil, A.R.M. (2015). Physiological Studies on Gladiolus Plant. M.Sc. Thesis, Fac. Agric. Minia Univ., Egypt.

Mazhar, A.A.M. and Eid, R.A. (2016). Effect of various doses of chemical fertilizer (kristalon) individually or in combination with different rates of biofertilizer on growth, flowering, corms yield and chemical constituents of Gladiolus grandiflorus. International Journal of Pharm Tech Research, 9(12):139-145.

Mirkalae, S.M.; Ardebili, Z.O. and Mostafavi, M. (2013). The effects of different organic fertilizations on the growth of Lilium longiflorum). Inter. Res. J. of Applied and Basic Sci., 4(1):181186.

Moghadam, A.R.; Ardebili, Z.O. and Saidi, F. (2012). Vermicompost induced changes in growth and development of Lilium asiatica hybrid var. Navona. African J. of Agric. Res., 7(17):26092621.

Mohamed, I.M.E. (2016). Effect of Some Physiological And Biological Treatments On The Growth, Flowering, Chemical And Anatomical Composition of Antirrhinum majus, L. Plant. Ph.D. Thesis, Fac. Agric., Cairo Univ., Egypt.

Mohammed, S.A.; Abd-Allatif, S.A. and Obaid, A.A. (2016). Effect of foliar application with potassium sulphate and ascorbic acid on growth and flowering of dahlia (Dahlia variabilis, L. cv. Arizona). Diyala for Agricultural Sciences Journal, 8(1):232-248.

MSTAT-C (1986). A Microcomputer Program for the Design Management and Analysis of Agronomic Research Experiments (Version 4.0), Michigan State Univ., U.S.A.

Munne-Bosch, S. and Algere, L. (2002). The function of tocopherol and tocotrienol in plants. Crit. Rev. Plant Sci., 21:31-57.

Pal, S.L. and Singh, D. (2019). Effect of integrated nutrient management (INM) on growth, flowering and yield in gladiolus (Gladiolus grandiflorus, L.) cv. White Prosperity. Asian Resonance Journal, 8(2):1-5.

Pandey, A.; Singh, A. and Sisodia, A. (2013). Effect of vermicompost and biocontrol agents on growth and flowering of gladiolus cv. J.V. Gold. The Asian Journal of Horticulture, 8(1):46-49.

Pandey, S.K.; Kumari, S.; Singh, D.; Singh, V.K. and Prasad, V.M. (2017). Effect of biofertilizers and organic manures on plant growth, flowering and tuber production of dahlia (Dahlia variabilis, 
L.) cv. S.P. Kamala. Int. J. Pure App. Biosci. 5(2):549-555.

Pansuriya, P.B.; Varu, D.K. and Viradia, R.R. (2018). Effect of biostimulants and biofertilizers on growth, flowering and quality of gladiolus (Gladiolus grandiflorus, L.) cV. American Beauty under greenhouse conditions. International Journal of Chemical Studies, 6(2):2191-2196.

Poursafarali, E.; Hashemabadi, D. Kaviani, B. and Kholdi, A. (2011). Effect of different cultivation beds on the vegetative growth of Polianthus tuberosa, L. Afr. J. Agric. Res., 6(19):4451-4454.

Prasad, L.; Saravanan, S.; Lall, D. and Singh, V.K. (2017). Effect of organic manure and inorganic fertilizer on plant growth and flower yield of Asiatic lily (Lilium longiflorum) sp. Zephyranthes. Environment \& Ecology J., 35(2A):929932.

Samoon, S.A.; Neelofar, A. and Aziz, M.A. (2019). Effect of organic and inorganic manure on vegetative parameters of
Alstroemeria under protected conditions. International Journal of Chemical Studies. 7(2):1964-1967.

Srivastava, R.; Preetham, S.P. and Chand, S. (2014). Effect of organic manures and biofertilizers on vegetative, floral and post-harvest attributes in tuberose (Polianthes tuberosa) var. Shringar. Asian J. Biol. Life Sci., 3(1):6-9.

Taha, R.A. and Hassan, A.H. (2008). Response of gladiolus plants to mineral and bio-fertilization treatments. 1Vegetative growth and flowering. Alex. J. Agric. Res., 53:79-86.

Tirkey, P.; Kullur, L.R. and Prasad, V.M. (2017). Effect of organic and inorganic source of N.P.K on growth and yield parameters of gladiolus (Gladiolus grandiflorus) cv. Jester. Journal of Pharmacognosy and Phytochemistry, 6(5):1004-1006.

Treder, J. (2008). The effects of coccopeat and fertilization on the growth and flowering of oriental lily "Star Gazer". J. of Fruit and Ornamental Plant Res., 16:361-370.

\title{
تأثير معاملات سماد المزرعة والتسميد الحيوي و/أو بعض الفيتامينات على والتى

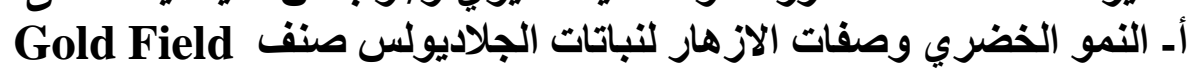

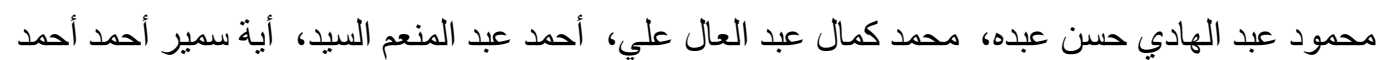

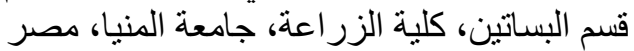

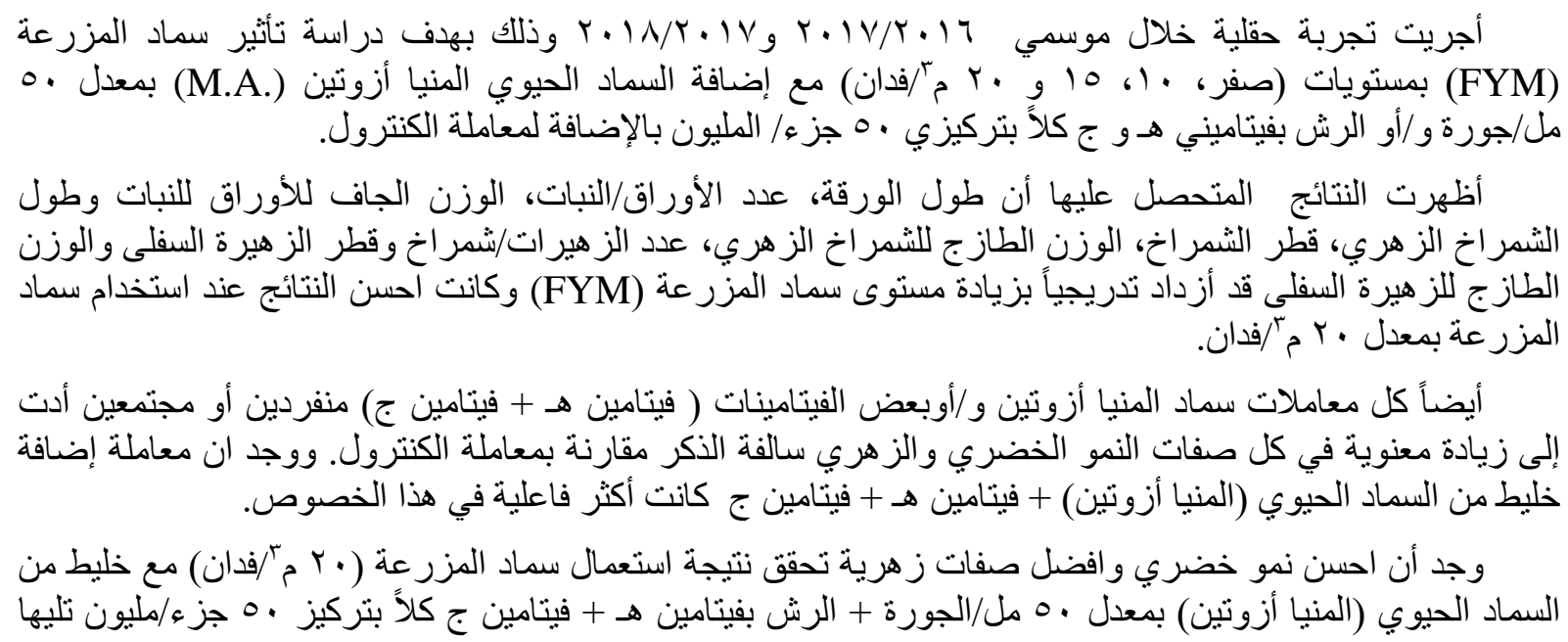




\section{M.A.H. Abdou et al.}

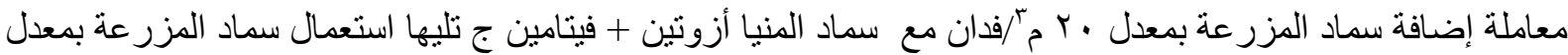

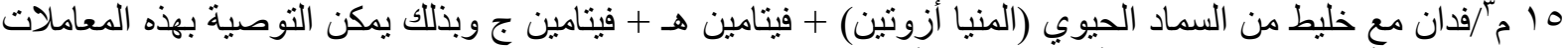
للحصول على أفضل نمو خضري من الفماد أفضل انتاج للأز هار من حيث الكم و الجودة. 